\title{
Retrograde amnesia following combined hippocampus-amygdala lesions in monkeys
}

\author{
DAVID P. SALMON, STUART ZOLA-MORGAN, and LARRY R. SQUIRE \\ Veterans Administration Medical Center and University of California, San Diego, California
}

\begin{abstract}
To assess the retrograde amnesia associated with medial temporal lesions, 8 cynomolgus monkeys were trained on object discrimination problems at different times prior to surgery. Different sets of 20 two-choice object discriminations were trained beginning $32,16,8,4$, and 2 weeks prior to surgery. Additionally, 6 weeks prior to surgery, all monkeys were trained on a motor skill task. Four monkeys then sustained bilateral removal of the hippocampus, amygdala, and overlying allocortex. The other 4 monkeys served as unoperated controls. Three weeks after surgery, memory for the 100 preoperatively learned object discriminations was assessed by presenting each of the object pairs for a single trial. The control group evidenced little forgetting of the discriminations: They performed as well on object pairs they had last seen 9 months earlier as on object pairs they had learned a few weeks earlier. The operated group demonstrated a profound loss of memory for all the discriminations: They performed at or near chance on the object pairs from all of the five preoperative time periods. Essentially the same results were obtained in four additional retention tests conducted during the following 8 months. Thus, retrograde amnesia was not temporally graded across the 8-month preoperative period sampled by the study. In contrast to these findings, retention of the preoperatively learned motor skill was excellent in both groups. Finally, additional tests showed that the learning of new object pairs was impaired after surgery, whereas a new motor skill was acquired at a normal rate.
\end{abstract}

Studies of human amnesia have been useful in addressing a number of questions about how memory is organized in the brain (Baddeley, 1982; Schacter, 1985; Squire, 1986; Weiskrantz, 1982). Amnesia consists of severe anterograde amnesia and some degree of retrograde amnesia, together with intact general intelligence, intact immediate memory, and intact language and social skills. The anterograde amnesia (deficient new learning ability) extends across all sensory modalities and across both verbal and nonverbal material. Nevertheless, the capacity for some kinds of learning is intact, most notably, skills and priming.

Retrograde amnesia, that is, the memory impairment for information acquired before the onset of amnesia, is quite variable. The noted amnesic patient H.M. (Scoville \& Milner, 1957), who sustained bilateral resection of the medial temporal lobe, including the hippocamus and amygdala, was reported to exhibit a temporally limited retrograde amnesia affecting 3 to 11 years prior to his surgery (Corkin, 1984; Marslen-Wilson \& Teuber, 1975). Patient R.B., who had amnesia associated with bilateral damage to the CAl field of the hippocampus (ZolaMorgan, Squire, \& Amaral, 1986), had little detectable retrograde amnesia, and at most the few years prior to

\footnotetext{
This research was supported by the Medical Research Service of the Veterans Administration and by NSF Grant BNS85-12349. We thank David Amaral for surgical and histological assistance and Carol Micheletti for research assistance. D.P.S. thanks Nelson Butters for providing support during the period of this investigation. Requests for reprints should be sent to Stuart Zola-Morgan, Veterans Administration Medical Center-San Diego, 3350 La Jolla Village Drive, San Diego, CA 92161.
}

the onset of amnesia were affected. Patients who have recovered from encephalitis also have medial temporal lobe damage (and frequently additional damage), but they vary greatly in the extent of retrograde amnesia (cf. Parkin, 1984). They can have temporally limited retrograde memory impairment (Rose \& Symonds, 1960) or a severe impairment involving several decades of the past (Cermak \& O'Connor, 1983). Patients with alcoholic Korsakoff's syndrome also exhibit extensive retrograde amnesia covering several decades (Butters, 1984; Squire \& Cohen, 1984; Weiskrantz, 1985).

One important conclusion about retrograde amnesia to emerge from studies of medial temporal amnesia (e.g., Patients H.M. and R.B.) is that very remote memory is preserved. Several researchers have proposed that an interaction must occur between the limbic system (the hippocampus, in particular) and sites of memory storage in neocortex in order for information to be represented in an enduring and accessible way (Halgren, 1984; Mishkin, 1982; Squire, 1987; Squire, Cohen, \& Nadel, 1984; Teyler \& DiScenna, 1986; Wickelgren, 1979). One view of this issue has been that: (1) Limbic structures remain involved with memory storage sites during some or all of a lengthy period of consolidation; (2) the role of these structures progressively diminishes as consolidation occurs; (3) memory storage sites eventually become independent of limbic structures, such that a lesion made sufficiently long after learning should have no effect on memory for what was learned (Squire, 1986, 1987).

It has been difficult to carry out a definitive test of this hypothesis. The idea is based largely on studies of hu- 
man patients, in whom memory for the remote past can be tested only imperfectly. In addition, the information available in some case reports is based only on material from clinical interviews. Even when objective remotememory tests are used, it is difficult to know when information was actually acquired by subjects or how strongly it was learned.

Studies of electroconvulsive shock (ECS) in rodents (Squire \& Spanis, 1984) and electroconvulsive therapy (ECT) in psychiatric patients (Squire, 1984) have shown that long-term memory is differentially vulnerable to disruption. For example, memory was severely disrupted by convulsive stimulation when it was 1 day old, partially disrupted when it was 1 to 3 weeks old, and unaffected when it was more than 3 weeks old (Squire \& Spanis, 1984). Thus, retrograde amnesia can be temporally graded across relatively long time periods, a finding consistent with the idea of a gradual and long-lasting consolidation process. However, the neuroanatomical basis of convulsive stimulation is unknown. It is still not entirely clear how medial temporal lesions affect past memories and whether such lesions can affect memories in a temporally graded fashion as a function of their age.

The recent establishment of an animal model of human amnesia in the monkey (Mahut $\&$ Moss, 1984; Mishkin, 1978; Squire \& Zola-Morgan, 1983) means that in principle retrograde amnesia can now be studied directly. Bilateral medial temporal lobe lesions produce severe anterograde amnesia across many kinds of memory tasks, while the ability to learn and retain skills is preserved (Mishkin, 1978; Zola-Morgan \& Squire, 1984, 1985). In the present study, we trained monkeys on a number of object-discrimination problems at different time periods prior to medial temporal surgery, and we tested postoperative retention of these problems. In this way we were able to control both the time at which information was initially acquired and the strength of the original learning. Previous work had shown that the object-discrimination problems used in the present study are sensitive to anterograde amnesia; that is, monkeys with medial temporal lesions are severely impaired in learning and remembering such problems (Zola-Morgan \& Squire, 1984, 1985).

\section{METHODS}

\section{Subjects}

Eight experimentally naive cynomolgus monkeys (Macaca fascicularis) served as subjects. They were assigned to two groups on the basis of their preoperative learning and retention scores. Four were used as normal controls $(\mathrm{N})$, and 4 received bilateral conjoint lesions of the hippocampus and amygdala $(\mathrm{H}-\mathrm{A})$.

\section{Surgery}

All surgery was performed in one stage under sodium pentobarbital anesthesia $(30 \mathrm{mg} / \mathrm{kg})$. Each monkey was anesthetized and placed in a stereotaxic instrument. A longitudinal midline incision was made, and the skin, fascia, and muscle were retracted bilaterally to expose the dorsal surface of the skull. Monkeys first sustained bilateral radio-frequency (RF) lesions of the amygdala, fol- lowed immediately by bilateral aspiration lesions of the hippocampus.

For the amygdala lesions, a small, rectangular, midline craniotomy was made, exposing the sagittal sinus approximately $18 \mathrm{~mm}$ in front of the external auditory meatus (instrumental zero in the atlas of Szabo \& Cowan, 1984). Two small, rectangular craniotomies centered at approximately $9 \mathrm{~mm}$ lateral to the sinus were then made on each side of the midline opening. The tip of the RF electrode was then positioned over one of the openings according to the anterior-posterior (AP) and medial-lateral (ML) atlas coordinates. Landmarks at the base of the cranium predict the position of forebrain structures such as the amygdala more accurately than stereotaxic coordinates taken relative to the auditory meatus (Aggleton, 1985; Szabo \& Cowan, 1984). Thus, the RF electrode was lowered to the base of the cranium, and the dorsal-ventral (DV) coordinates for the amygdala lesion were then calculated relative to the base of the cranium. Four lesions of the amygdala were made on each side of the brain. Separate lesions were made in the dorsal (AP 18.5; ML 9.5; DV -4.0) and in the ventral (AP 18.5; ML 9.5; DV -6.5) portions of the anterior extent of the amygdala, and separate lesions were made in the dorsal (AP 17.0; ML 9.0; DV -4.0) and ventral (AP 17.0; ML 9.0; DV -6.5) portions of the posterior extent of the amygdala. A Grass RF lesion maker (Model RF-1) was used in combination with a Biotronics lesion electrode (Model E-1), insulated except for a 3-mm tip. At each coordinate, the tip of the electrode was brought to a temperature of approximately $80^{\circ} \mathrm{C}$ for $45 \mathrm{sec}$.

When the amygdala lesions were completed, the monkeys were transferred to a specially designed headholder that permitted unobstructed access to the temporal portion of the head. The temporal muscles on each side were fully retracted, the zygomatic arch was removed, and openings were made on each side of the skull to expose the lateral-ventral portion of the temporal lobe of the brain. The hippocampus on each side was then approached by elevating the occipitotemporal convexity and entering the brain through the entorhinal cortex at a point medial to the occipitotemporal sulcus. A small-gauge glass pipette attached to a variable-intensity suction pump was used to aspirate the entorhinal cortex and much of the parahippocampal gyrus (Area TF-TH of Bonin \& Bailey, 1947). The exposed hippocampus was then removed by aspiration with the upper surface of the lateral ventricle being used as an identifiable dorsal boundary along the entire length of the removal. In this way it was possible to spare the temporal stem during surgical removal of the hippocampus (Zola-Morgan, Squire, \& Mishkin, 1982). The resulting bilateral lesion was thus intended to reproduce the conjoint hippocampal-amygdala removal achieved in previous studies (Zola-Morgan \& Squire, 1984, 1985; Zola-Morgan et al., 1982). Following achievement of hemeostasis, the dura, muscle, fascia, and skin were sutured. The monkeys were placed in a specially designed incubator for 24 to $48 \mathrm{~h}$ following surgery and were then returned to their home cages.

\section{Behavioral Testing}

Preoperative training: Pretraining and practice problems. All testing was carried out in a Wisconsin General Test Apparanus (WGTA; Harlow, 1944). During four to six sessions of pretraining, all monkeys were taught to obtain food by displacing objects that covered either of two food wells. Upon completion of pretraining, the monkeys were given two practice problems, in succession on the same day, to prepare them for the main part of the experiment described below. The practice problems were two separate two-choice discrimination tasks consisting of pairs of objects that could be learned by normal monkeys in a single session (ZolaMorgan \& Squire, 1984). These objects, as well as the objects used during the main experiment, were taken from a pool of more than 300 junk objects. The practice problems were administered with an intertrial interval of $15 \mathrm{sec}$; the monkeys were trained to a learn- 
ing criterion of 18 correct responses in 20 trials. A raisin reward was always concealed under the correct object. The position of the correct object over the left or the right food well varied from trial to trial according to a random sequence (Gellerman, 1933). The main experiment began on the day after the practice problems had been learned.

Preoperative training: Discrimination pairs. An outline of the experimental design is presented in Table 1 . At each of five time periods prior to surgery, all 8 monkeys learned 20 two-choice objectdiscrimination problems. Specifically, a different set of 20 object pairs was trained beginning 8 months, 4 months, 2 months, 1 month, and approximately 2 weeks ( 12 days) prior to surgery. The 20 object pairs of each set were presented in the same order to each monkey. Each set of 20 problems was administered during a 10-day period, 2 problems per day, and each object-pair problem was trained to a criterion of 18 correct responses in 20 trials. The intertrial interval was $15 \mathrm{sec}$. In addition, a retention test was administered $24 \mathrm{~h}$ after presentation of the last pair in each 20-pair set. For the retention test, each of the 20 pairs was presented once in a random order during a single session. In this way, the monkeys learned a total of 100 object pairs prior to surgery, and retention was tested 1 day after the learning of each 20-pair set.

The performance scores on the five 24-h retention tests were used to divide the monkeys into two matched groups of 4 monkeys each. The monkeys in one group received bilateral conjoint $\mathrm{H}-\mathrm{A}$ lesions. The monkeys in the other group served as unoperated controls. Surgery was performed $24 \mathrm{~h}$ after the preoperative retention test of the fifth and final training period.

Preoperative training: Motor skill task. The monkeys also learned a motor skill task (the Lifesaver task) prior to surgery, as adapted from one described by Davis, McDowell, Deter, and Steele (1956; see Zola-Morgan \& Squire, 1984). The Lifesaver task was given between the fourth and fifth 20-pair set, about 3 weeks prior to surgery. The apparatus consisted of a horizontally oriented metal rod with a right-angle bend. With the opaque door of the WGTA lowered, a candy Lifesaver was threaded onto the rod. The opaque door was then raised, and the monkey was allowed $30 \mathrm{sec}$ to retrieve the Lifesaver by maneuvering it around the bend and off the rod. If $30 \mathrm{sec}$ elapsed before the monkey obtained the Lifesaver, the opaque door was lowered and the trial was reset with the Lifesaver at the starting point. Six trials were administered daily for 8 consecutive days.

Postoperative retention tests. Following surgery and a 3-week recovery period, retention of all 100 preoperatively learned object pairs ( 20 pairs $\times 5$ preoperative time periods) was assessed in both the operated monkeys and the normal control monkeys. In two daily sessions of 50 trials each, all 100 pairs were presented once. The order of presentation of the 100 pairs was random, with the constraint that an equal number of pairs from each time period was presented on each day. Presenting each of the 100 pairs for only a single trial provided a measure of retention that was not influenced by the ability to relearn the discriminations.

We next assessed retention of the preoperatively acquired Lifesaver motor skill. At 4 weeks after surgery, the monkeys were given one daily session of six trials. Then, at 8 weeks after surgery, they were given two additional daily sessions.

We also evaluated postoperative retention of the 100 preoperatively learned object pairs on four additional occasions over the next 8 months. Three tests of retention and one test of relearning ability were used: (1) At 8 weeks after surgery, we repeated the retention test described above to determine whether any recovery of preoperatively acquired memory had occurred in the operated monkeys. (2) At 11 weeks after surgery, we added a procedure to ensure that the operated monkeys were familiar with the rules of discrimination testing and to increase the probability that these rules would be used during the postoperative retention test. Accordingly, the retention test was repeated in an identical way, but it was preceded on each of the 2 test days by training on two new objectdiscrimination problems to a criterion of 18 correct responses in 20 trials. (3) At 26 weeks after surgery, we sought to increase the sensitivity of the retention test. The retention test was therefore administered in the same way as described above, except that a threechoice retention test was given. The three-choice retention test involved presenting one of the 100 originally correct objects together with two originally incorrect objects. For this test, each of the 100 incorrect objects was used twice, with the constraint that an incorrect object appeared only once on each of the 2 test days. (4) At 29 weeks after surgery, we evaluated retention of the preoperatively learned pairs in a different way, by determining the ability of the animals to relearn them. In humans, relearning ability provides a particularly sensitive index of memory for recently learned material (Nelson, 1978). Thirty discrimination pairs from the original 100 pairs, 6 from each of the five time periods, were trained to a criterion of 9 correct trials in 10 . The order of presentation of the 30 pairs was random, with the constraint that pairs from each of the five time periods were evenly distributed through the sequence. Three or four pairs were trained each day.

Postoperative tests of new learning ability. Postoperative learning ability was assessed during the week after the final test of retrograde amnesia. In total, eight new object-discrimination problems were presented. None of these objects had been used previously. On the 1st day, four object pairs were trained to a learning criterion of 9 correct trials in 10 , and retention was tested 5 days later by presenting each pair for 1 trial. Then four additional object pairs were trained to the same criterion, and retention was similarly tested 5 days later.

The monkeys were also tested postoperatively for their ability to learn and retain a new motor skill. The monkeys were trained

Table 1

Outline of retrograde amnesia study

\begin{tabular}{lccccccc}
\hline & Training & & \multicolumn{3}{c}{ Surgery } & \multicolumn{2}{c}{ Testing } \\
\hline $8 \mathrm{mo}$ & $4 \mathrm{mo}$ & $2 \mathrm{mo}$ & $1 \mathrm{mo}$ & $2 \mathrm{wk}$ & $\begin{array}{c}3-\mathrm{wk} \\
\text { recovery }\end{array}$ & $\begin{array}{c}\text { Retention of } \\
\text { preoperatively } \\
\text { learned problems }\end{array}$ & $\begin{array}{c}\text { Learning of } \\
\text { new problem }\end{array}$
\end{tabular}

Learn Learn Learn Learn Learn

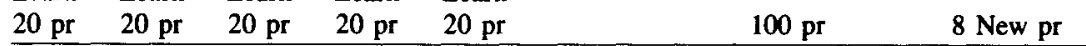

Note-Abbreviations: $\mathrm{mo}=$ months; $w \mathrm{k}=$ weeks; $\mathrm{pr}=$ pairs, or two-choice object problems. At each of five time periods prior to surgery, all monkeys learned 20 two-choice objectdiscrimination problems. Two problems per day were administered for 10 days, and a different set of 20 object pairs was presented at each time period. On the day after the monkeys learned the 20th object pair of each set, retention of the entire set was assessed by presenting each of the 20 pairs for a single trial. Following surgery, retention of all 100 preoperatively learned pairs was assessed by presenting each of the 100 pairs for a single trial. Finally, the ability to learn and remember 8 new pairs was assessed. 
to reach through a hole in a clear plastic vertical barrier in order to obtain a candy Lifesaver. The Lifesaver could be removed from a 1 -in.-long vertical peg mounted 2 in. above and 1 in. behind the hole. The peg was located so that the monkeys had to maneuver the Lifesaver up from the base of the peg, using only two fingers. Six trials per day were given, each with an upper limit of $60 \mathrm{sec}$. If the monkeys did not obtain the Lifesaver within $60 \mathrm{sec}$, the opaque door was lowered to terminate the trial. Retention of the skill was evaluated in one six-trial session 1 month after the final day of training. One monkey in each group was unable to learn this task and was excluded from the data analysis.

\section{RESULTS}

\section{Histological Findings}

The brains of the 4 operated monkeys were fixed in sugar/formalin and embedded in albumin. Frozen sections were cut at $50 \mu$, and every fifth section was stained with thionin. All 4 monkeys sustained extensive bilateral removals of the amygdala and the hippocampus (Figure 1). Removal of the amygdala in 3 monkeys (Monkeys H-A 1, H-A 2, and H-A 4) was nearly complete bilaterally, with the exception that Monkey H-A 1 evi- denced sparing of the mediodorsal portion of the amygdala on the left side in the region of the cortical and medial nuclei. The other monkey (Monkey H-A 3) sustained considerable bilateral damage of the amygdala, but damage was limited to its ventromedial portion; almost all of the central nucleus and the medial nucleus were spared bilaterally. With the exception of the most posterior 2-3 mm, the entire hippocampus was removed in 3 monkeys. In the remaining monkey (Monkey H-A 4), removal of the hippocampus was complete on one side; on the other side, the posterior third of the hippocampus was spared.

In addition, the lesions in all 4 monkeys included bilaterally all the allocortex of the entorhinal area (with the exception of Monkey H-A 2, in which the anterior half of the entorhinal cortex was spared unilaterally), and over 90\% of the parahippocampal gyrus (with the exception of Monkey H-A 2, in which the posterior half of the parahippocampal gyrus was spared bilaterally, and Monkey H-A 1 , in which the posterior third of the parahippocampal gyrus was spared unilaterally). Some damage to inferotemporal cortex (Area TE, Bonin \& Bailey, 1947) occurred in all 4 animals. In 2 of the animals (Monkeys

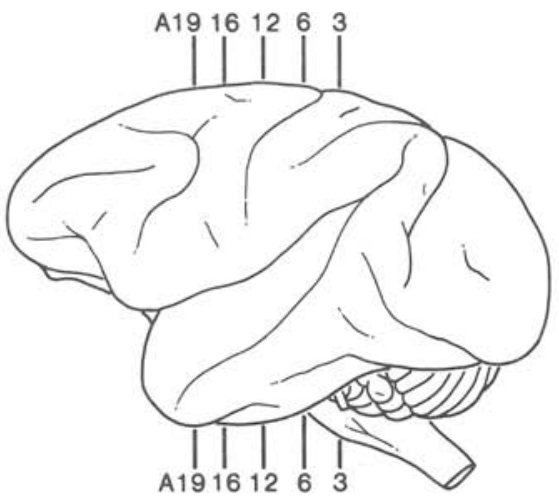

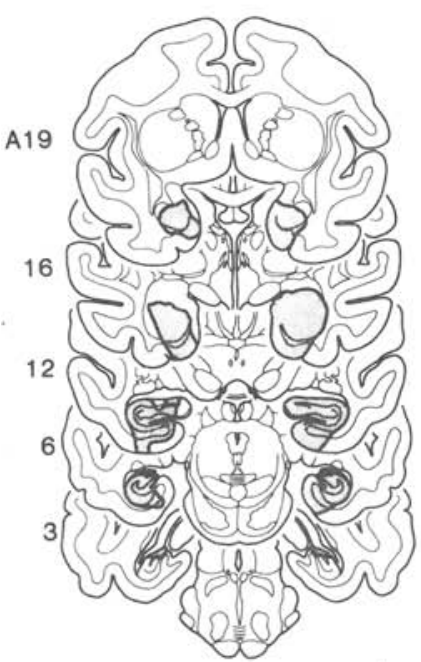

H-A 1

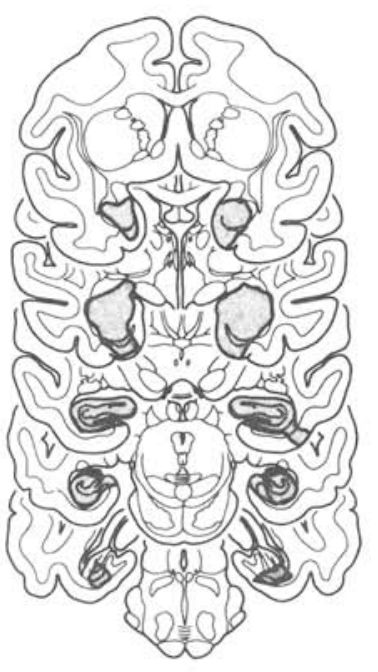

H-A 2

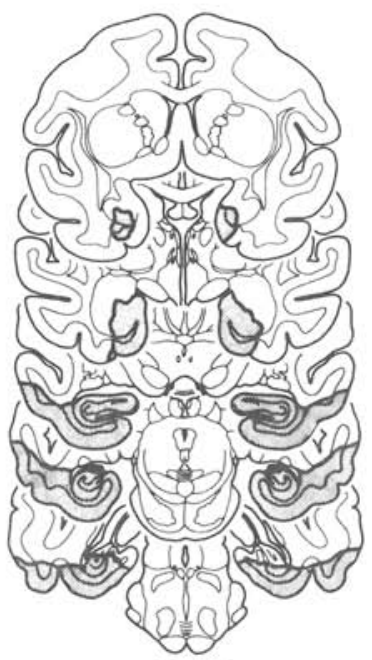

H-A 3

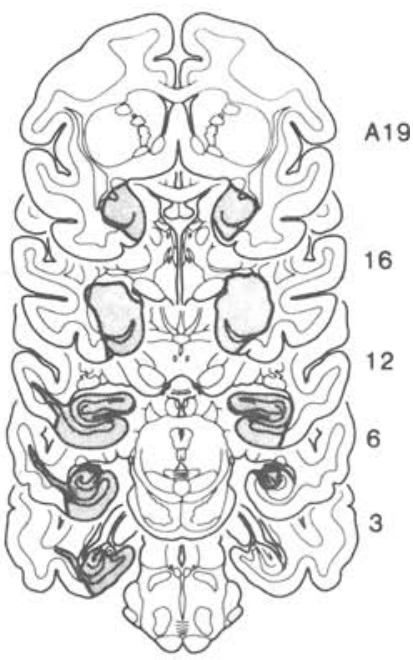

$\mathrm{H}-\mathrm{A} 4$

Figure 1. Representative coronal sections through the temporal lobes, showing the extent of damage (shaded region) in the 4 monkeys with combined hippocampus-amygdala (H-A) lesions. Numerals adjacent to coronal sections indicate the approximate distance (in millimeters) from the interaural plane and correspond to those on the lateral view. (Atlas drawings based on Szabo \& Cowan, 1984.) 
$\mathrm{H}-\mathrm{A} 1$ and $\mathrm{H}-\mathrm{A} 2$ ), the damage involved less than $15 \%$ of Area TE bilaterally. This damage probably resulted from inadvertent direct mechanical pressure applied during the elevation of the occipitotemporal convexity. In Monkey H-A 3, the ventral half of the inferotemporal gyrus was damaged bilaterally for most of its anteriorposterior extent (presumably as a result of a surgically related infarct). In Monkey H-A 4, there was extensive gliosis unilaterally in the subcortical white matter of the inferotemporal cortex in a band stretching from the ventricle laterally into the inferotemporal gyrus. There was no relationship between the severity of behavioral impairment on any of the five object-retention tests and the extent of damage to Area TE in the monkeys with lesions of the medial temporal region.

In 2 monkeys (Monkeys H-A 2 and H-A 4), the caudate nucleus was damaged on one side. Neither the lateral geniculate nuclei nor the temporal stem sustained noticeable damage in any of the animals. The medial dorsal nucleus of the thalamus appeared normal. There was no evidence of cell loss in the mammillary nuclei, but there was gliosis in the medial mammillary nucleus at the site of termination of the fornix. Extensive gliosis was observed throughout the fornix.

\section{Behavioral Findings}

Preoperative training: Discrimination pairs. The monkeys were able to learn the 20 object pairs of the first set (given at 32 weeks prior to surgery) in an average of 3.3 to 8.6 trials (not including the run of 18 correct of 2 criterion trials). Subsequent objects sets were learned more quickly (range $=0.1$ to 2.8 trials for individual monkeys). Learning of the five sets of 20 object pairs was comparable for the 4 monkeys that subsequently underwent surgery (H-A) and for the 4 normal control monkeys (mean trials to criterion for the 20 object pairs learned 32 weeks prior to surgery: $\mathrm{H}-\mathrm{A}=4.84, \mathrm{~N}=$ 4.95; trials to criterion for the four subsequent sets of 20 object pairs: $\mathrm{H}-\mathrm{A}=.61$ to 1.5 trials, $\mathrm{N}=.63$ to .95 trials).

Figure 2 shows performance on the five retention tests that were administered preoperatively, $24 \mathrm{~h}$ after completion of training on each set of 20 object pairs. Data are presented separately for the 4 monkeys that subsequently underwent surgery (H-A) and for the 4 normal control monkeys $(\mathrm{N})$. Both groups demonstrated good retention of the discriminations: They performed at approximately $80 \%$ correct on each of the five retention tests. A two-way analysis of variance showed that the two

\section{PREOPERATIVE RETENTION TEST}

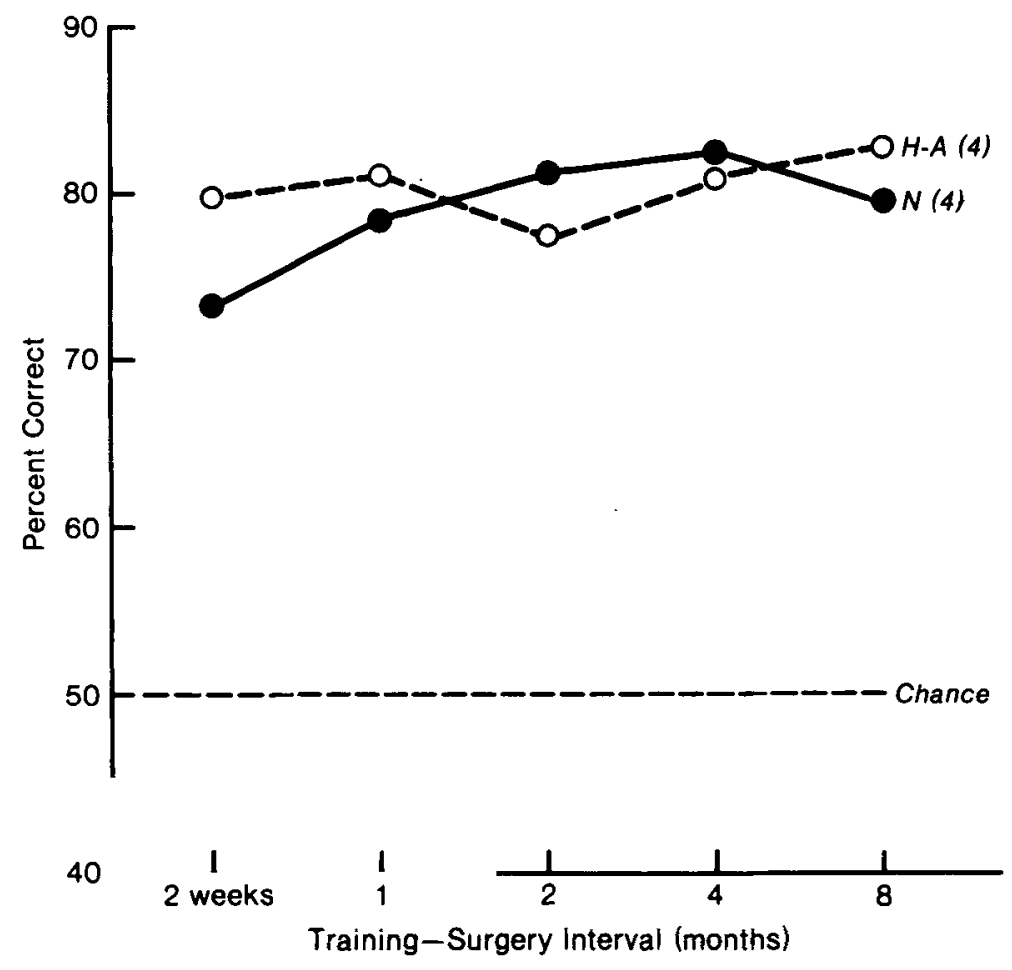

Figure 2. Retention of five different sets of 20 object pairs, assessed preoperatively, $24 \mathrm{~h}$ after the training of each set. Twenty pairs were learned at each of the indicated times prior to surgery, and retention was assessed by presenting each of the 20 object pairs for a single trial. $\mathbf{N}=$ normal control monkeys; $\mathbf{H}-\mathbf{A}=$ monkeys with bilateral removal of the hippocampus and amygdala. Numbers in parentheses show the number of monkeys in each group. 
groups performed similarly and that, within each group, retention scores were similar for the five sets of $20 \mathrm{ob}$ ject pairs [group, $F(1,6)=.10, p>.10$; set, $F(4,24)$ $=.26, p>.10$; group $\times$ set, $F(4,24)=.30, p>.10$ ]

Postoperative retention tests. Figure 3 shows the results of the first retention test, given 3 weeks after surgery. This test measured retention for 100 object pairs that had last been seen by the monkeys during one of five time periods, from 3 weeks to almost 9 months earlier. The normal control monkeys evidenced little forgetting of the discrimination problems across this time period, and there were no differences in the retention scores as a function of time period $[F(4,12)=2.21, p>.10]$. In fact, the normal monkeys obtained approximately the same percentage-correct scores on each 20-pair set as they had achieved on the preoperative retention tests $[F(1,3)$ $=6.2, p>.05]$.

In contrast to the good retention of the normal monkeys, monkeys with $\mathrm{H}$-A lesions demonstrated marked retrograde amnesia. The monkeys with $\mathrm{H}$-A lesions performed significantly worse postoperatively than they had preoperatively $[F(1,3)=46.5, p<.01]$. Moreover, with the exception of the 20-pair set administered beginning 2 weeks prior to surgery, the postoperative retention scores were no better than would have been expected by chance [for the object set administered 2 weeks prior to surgery: $t(3)=9.00, p<.01$; for all other sets, $t \mathrm{~s}$ ranged from .77 to $2.33, p s>.10]$. Overall, the postoperative retention scores of the H-A monkeys averaged $53 \%$, which was not significantly above chance $[t(3)$ $=1.53, p>.10]$. Finally, a two-way analysis of variance showed that the monkeys with $\mathrm{H}-\mathrm{A}$ removals performed significantly worse than the normal control monkeys did on the 20-pair sets [group, $F(1,6)=7.28, p<$ .05 ; set, $F(4,24)=3.40, p<.05$; group $\times$ set, $F(4,24)$ $=3.30, p<.05]$. The significant interaction term was due to the results obtained at the 4-month training-surgery interval, not to any systematic trend across the five time periods [analysis of variance without the 4-month data points: group $\times$ set, $F(3,18)=.60, p>.10]$.

Four additional tests of retention were administered between 8 and 29 weeks after surgery (Figure 4). At 8 weeks after surgery, when the retention test just described was repeated, the results were similar (Figure 4A). A twoway analysis of variance indicated a significant effect of group $[F(1,6)=38.6, p<.01]$, but no effect of set $[F(4,24)=1.4, p>.10]$ and no group $\times$ set interaction $[F(4,24)=0.4, p>.10]$. None of the five scores obtained by the $\mathrm{H}-\mathrm{A}$ group was significantly above chance ( $t \mathrm{~s}<2.0, p \mathrm{~s}>.10$ ), although the overall group mean

\section{RETENTION TEST 3 WEEKS AFTER SURGERY}

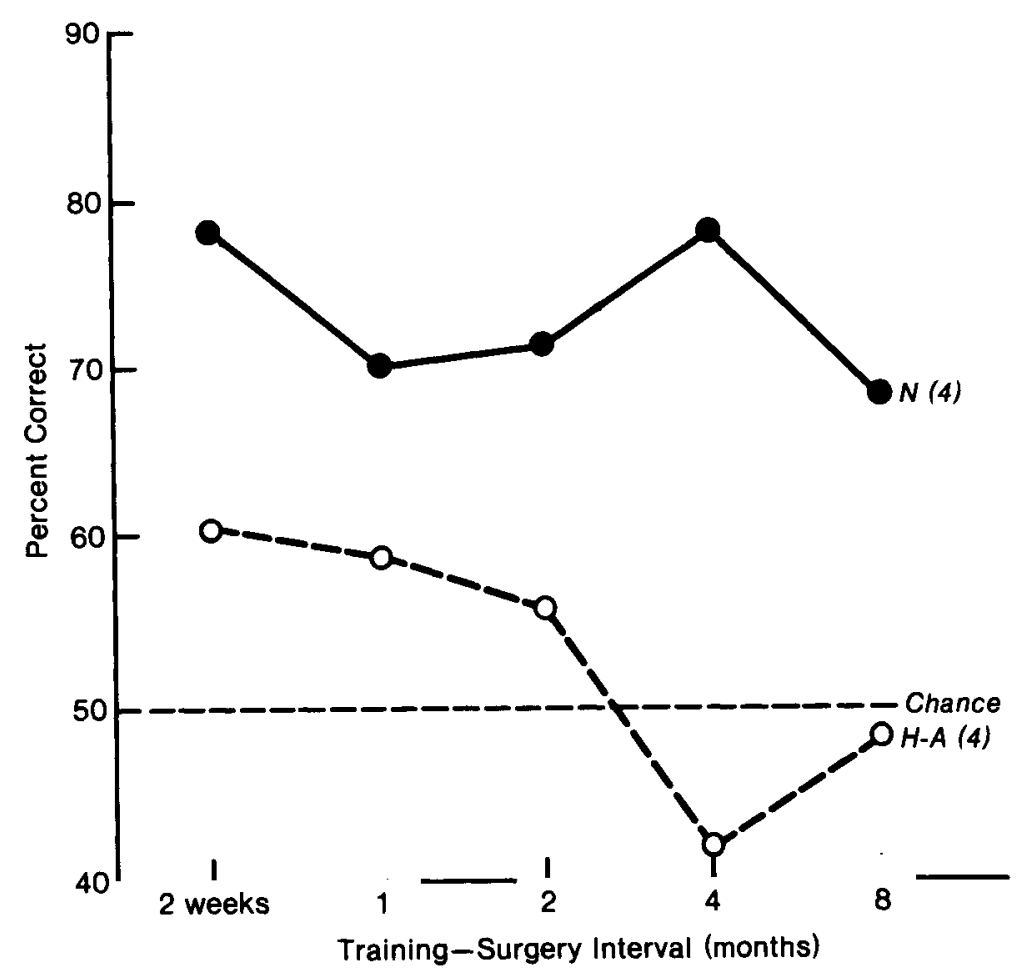

Figure 3. Retention of five different sets of 20 object pairs, learned at five different intervals prior to surgery, and assessed 3 weeks after surgery. Retention of all $100 \mathrm{ob}-$ ject pairs was assessed by presenting each pair for a single trial. $\mathbf{N}=$ normal control monkeys; H-A = monkeys with bilateral removal of the hippocampus and amygdala. Numbers in parentheses show the number of monkeys in each group. 

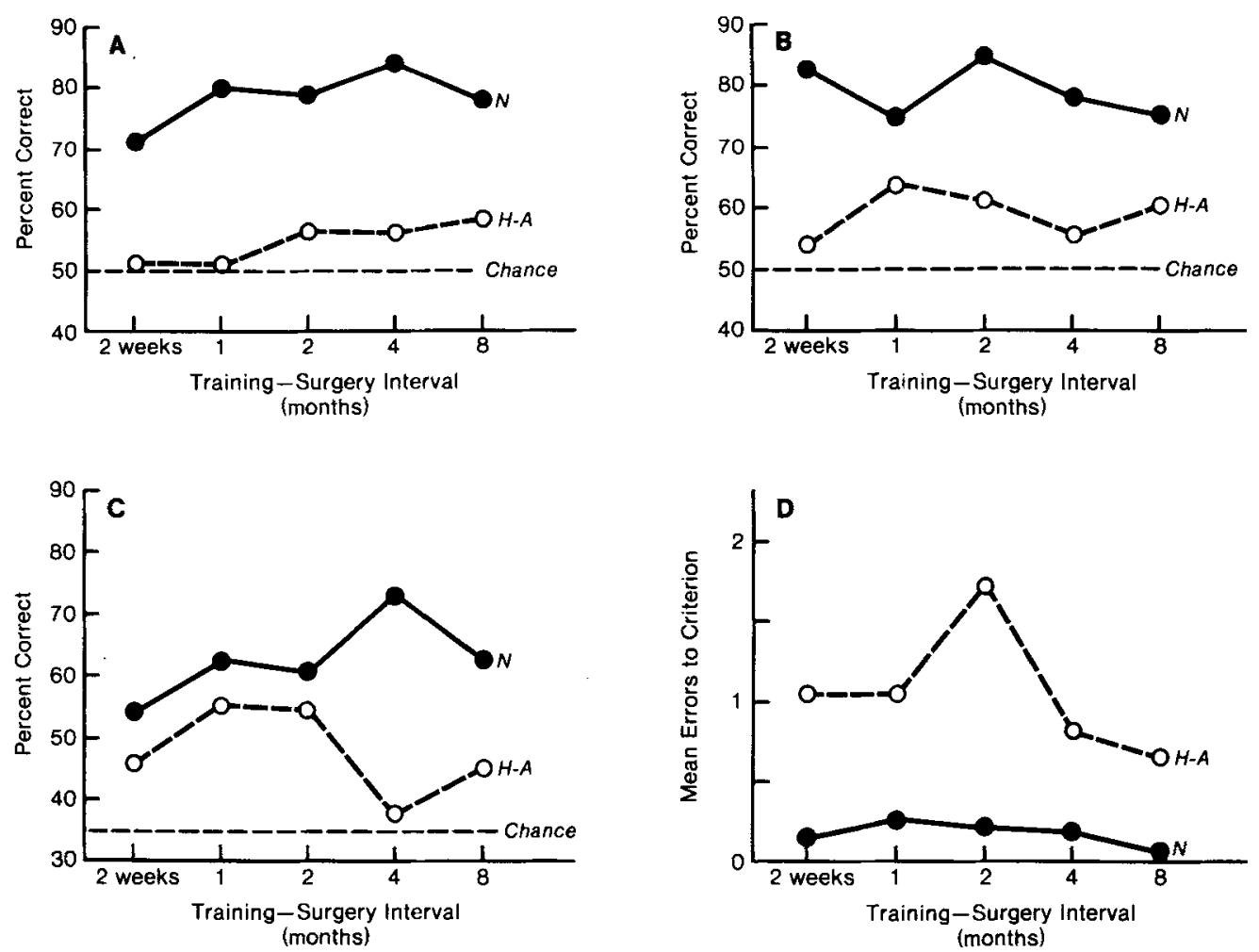

Figure 4. (A) Retention of five sets of 20 object pairs, learned at five intervals before surgery and assessed 8 weeks after surgery. Retention of all 100 object pairs was assessed by presenting each pair for a single trial. (B) Retention of the same five sets of 20 object pairs at 11 weeks after surgery. Each retention test was preceded by training on two new object pairs. (C) Retention of the same five sets of 20 object pairs at 26 weeks after surgery; a three-choice, instead of a two-choice, test was used. (D) Relearning of $\mathbf{3 0}$ of the 100 preoperatively learned pairs ( 6 from each of the five time periods). $N=4$ normal control monkeys; $H-A=4$ monkeys with bilateral removal of the hippocampus and amygdala.

$(54.3 \%)$ did reach significance $[\mathrm{t}(3)=3.83, p<.05]$. At 10 weeks after surgery, when each day's retention test was preceded by training on two new object pairs, the results were also similar (Figure 4B). A two-way analysis of variance indicated a significant effect of group $[F(1,6)=9.0, p<.05]$, no effect of set $[F(4,24)=1.0$, $p>.10]$, and a group $\times$ set interaction $[F(4,24)=3.1$, $p<.05]$. Within the H-A group, only one score (for the 1-month training-surgery interval) was significantly above chance $[t(3)=4.2, p<.05$; all other $t$ s $<2.6]$. The overall score for the H-A group (59\%) failed to reach significance $[t(3)=2.29, p>.10]$.

Increasing the sensitivity of the retention test by using a three-choice retention test also produced similar results (Figure 4C). The monkeys with $\mathrm{H}-\mathrm{A}$ lesions continued to demonstrate substantial retrograde amnesia, which appeared to extend across all five preoperative time periods. A two-way analysis of variance indicated a significant effect of group $[F(1,6)=4.78, p<.06]$, no effect of set $[F(4,24)=.65, p>.10]$, and a group $\times$ set interaction $[F(4,24)=4.15, p<.01]$. Within the H-A group, two scores (for sets trained 1 and 2 months before surgery) were significantly above chance $[t \mathrm{~s}(3)>3.2, p<.05$; all other $t \mathrm{~s}<1.9$ ]. The overall score for the H-A group
(47.5\%) was significantly above the chance value of $33 \%$ $[t(3)=3.4, p<.05]$. Thus, on three separate retention tests (Figure 4, A-C), the severity of retrograde amnesia demonstrated by the operated monkeys resembled what was found on the first retention test (Figure 3). The H-A monkeys were markedly impaired, sometimes performing close to chance levels for all five preoperatively learned 20-pair sets. Moreover, they exhibited no signs of a temporal gradient of retrograde amnesia, even in those cases in which the overall retention score was significantly above chance (Figures $4 \mathrm{~A}$ and $4 \mathrm{C}$ ).

Figure 4D presents the data for the 30 discrimination pairs (6 pairs randomly selected from each of the five object sets), which were relearned at 29 weeks after surgery. The monkeys with $\mathrm{H}-\mathrm{A}$ removals made significantly more errors before achieving criterion performance than did the normal monkeys $[F(1,6)=5.86, p=.05]$. There was no effect of set $[F(4,24)=2.10, p>.10]$, and no significant group $\times$ set interaction $[F(4,24)=1.49, p>$ $.10]$. The results were similar when analyzed on the basis of trials to criterion, except that the effect of set approached significance $[F(4,24)=2.18, p=.08]$. This finding-that is, no temporal gradient of retrograde amnesia-was obtained despite the fact that the method 
of relearning proved to be a sensitive measure of retention. Specifically, the 30 preoperatively trained object pairs were learned more quickly than the 8 new pairs (Figure 5). H-A monkeys relearned the 30 preoperatively trained object pairs with an average of 1.1 errors per pair (and 1.9 trials), whereas they required 2.5 errors (and 4.5 trials) to learn 8 new pairs [for errors, $t(3)=3.6$, $p<.05$; for trials, $t(3)=2.34, p=.10$ ]. Thus, with the relearning method it was possible to demonstrate that H-A monkeys retained a significant amount of preoperatively acquired information.

Figure 5 also shows that normal monkeys made fewer errors than did monkeys with $\mathrm{H}-\mathrm{A}$ lesions while relearning the 30 preoperatively trained object pairs [normal monkeys $=.1$ errors, $\mathrm{H}$-A monkeys $=1.1$ errors; $t(6)$ $=2.5, p<.05]$. The normal monkeys were also better at learning the 8 new object pairs (normal monkeys = .8 errors, H-A monkeys $=2.5$ errors), although this difference did not reach significance $[t(5)=1.95, p=$ $0.1]$. For this comparison, only 3 of the original 4 normal monkeys were available for testing. There was only one overlap between the scores of the 3 normal and the $4 \mathrm{H}$-A monkeys. On the retention test, which occurred 5 days after learning the 8 new object pairs, normal monkeys also made fewer errors (mean $=0$ ) than monkeys with $\mathrm{H}$-A removals [mean $=.31 ; t(5)=2.21, p<.08$ ] .

Preoperative training and postoperative retention: Motor skill task. Figure 6A shows preoperative acqui- sition of the motor skill task at 6 weeks prior to surgery for the 4 monkeys that subsequently underwent surgery (H-A) and for the 4 normal control monkeys (N). There were no significant differences in the preoperative learning scores $[F(1,7)=2.25, p>.10]$. There was a significant effect of session $[F(7,42)=24.89, p<.01]$ and no group $\times$ session interaction: $F(7,42)=1.84, p>$ $.10]$. Postoperative retention of the skill is shown in Figure 6B. In the first test, 4 weeks after surgery, the monkeys were given a single six-trial session. Two of the monkeys that had received hippocampus-amygdala lesions appeared to be apraxic in their home-cage behavior following surgery. These 2 monkeys showed the poorest performance on the postoperative retention test. Nevertheless, the retention scores of the normal and operated groups were not significantly different $[t(6)=1.61$, $p>$.10]. One month later, the monkeys were again tested for retention of the motor skill, this time for two daily sessions of six trials each. Both groups exhibited good retention of the task, and postoperative performance was similar to the level that had been achieved on the final three sessions of preoperative training.

Figure 7 shows learning (Figure 7A) and retention (Figure 7B) of the second motor skill task, the peg Lifesaver task, which was trained postoperatively. There were no significant differences between the normal monkeys and the monkeys with $\mathrm{H}-\mathrm{A}$ removals on the acquisition of the peg task. Asymptotic performance was reached by both

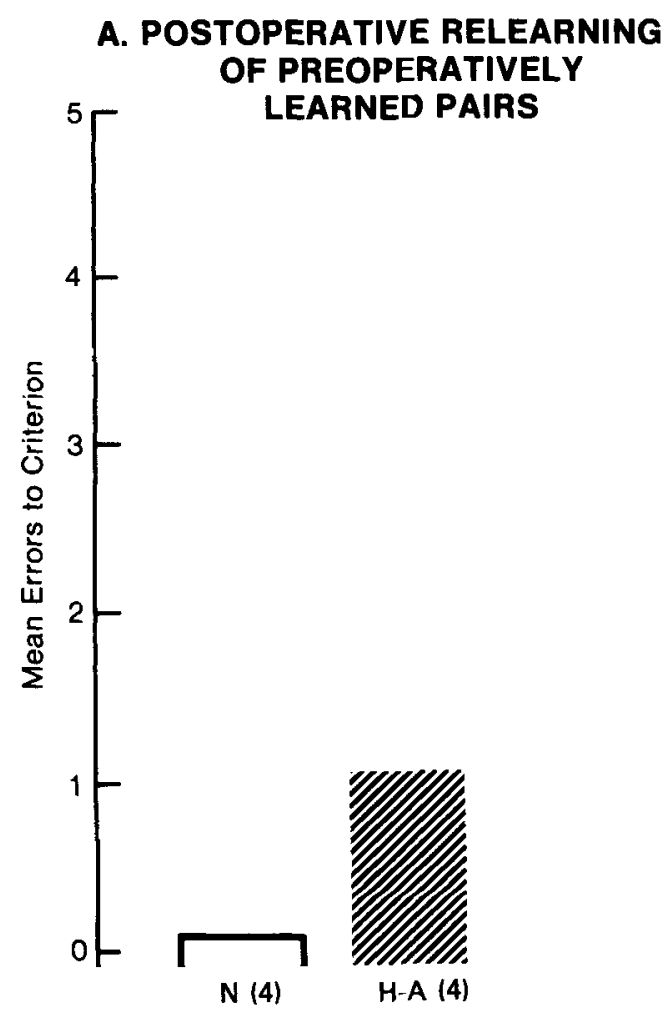

\section{B. POSTOPERATIVE LEARNING OF NEW PAIRS}

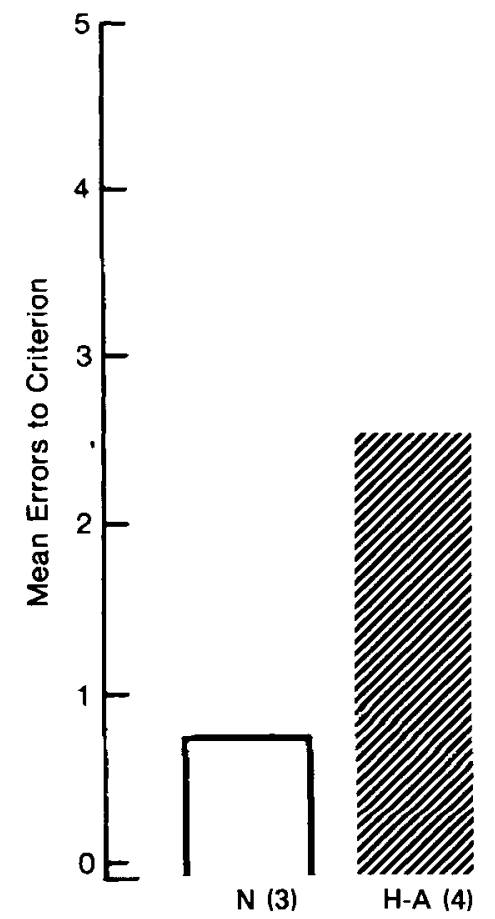

Figure 5. (A) Postoperative relearning of 30 preoperatively trained object pairs. (B) Postoperative learning of 8 new object pairs. $\mathrm{N}=$ normal control monkeys; $\mathrm{H}-\mathrm{A}=$ monkeys with bilateral removal of the hippocampus and amygdala. Numbers in parentheses show the number of monkeys in each group. 
A. PREoperative training

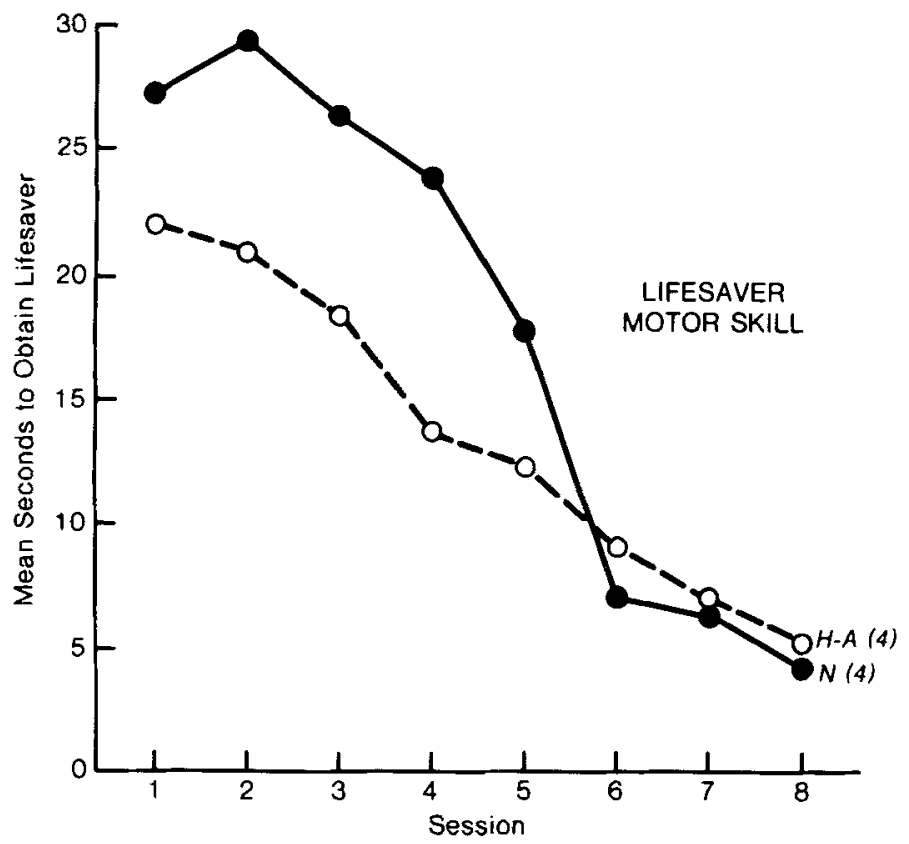

B. POSTOPERATIVE RETENTION

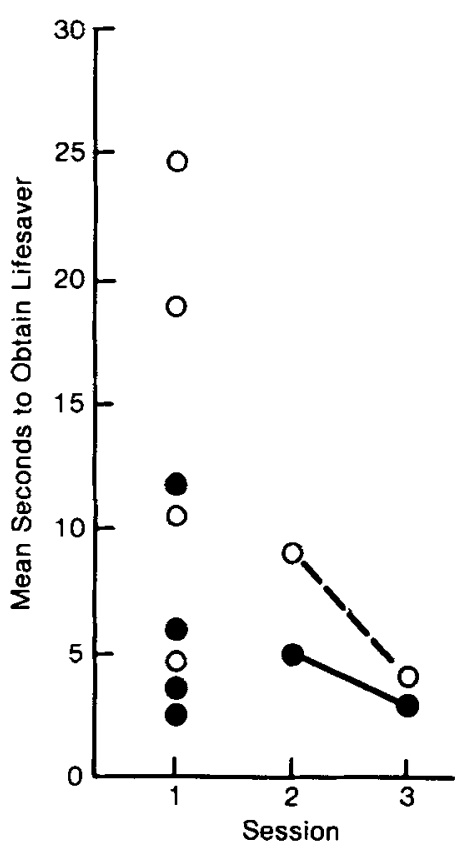

Figure 6 (A) Preoperative learning of a motor skill during eight daily sessions and (B) retention of the skill 4 to 8 weeks after surgery. $\mathrm{N}=$ controls; $\mathrm{H}$-A $=$ monkeys with bilateral removal of the hippocampus and amygdala. Numbers in parentheses show the number of monkeys in each group.

\section{A. LEARNING 16 WEEKS AFTER SURGERY}

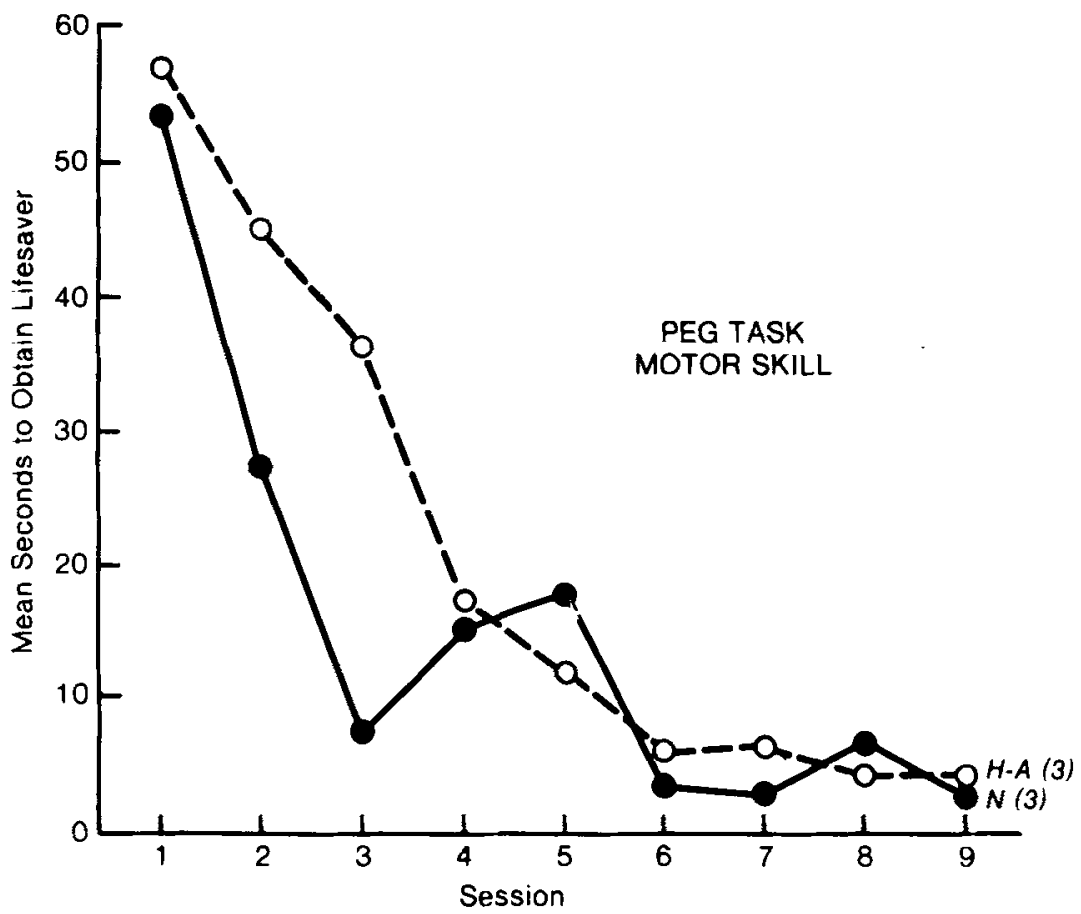

B. RETENTION

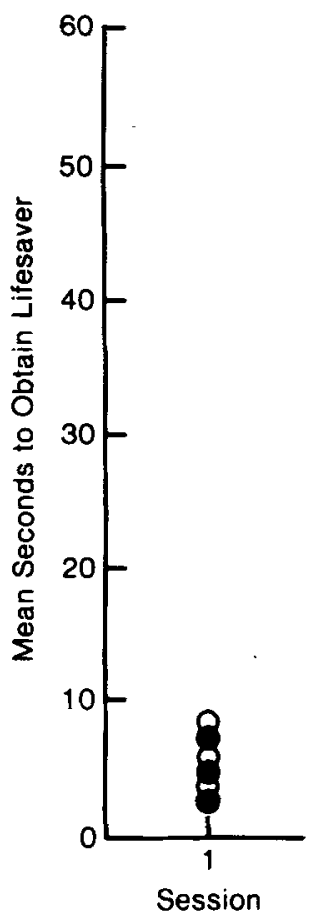

Figure 7. (A) Postoperative learning of a motor skill and (B) retention 1 month later. $\mathbf{N}=$ normal control monkeys; H-A = monkeys with bilateral removal of hippocampus and amygdala. Numbers in parentheses show the number of monkeys in each group. 
groups on Day 6 [two-way analysis of variance; group: $F(1,4)=2.08, p>.10$; session: $F(8,32)=25.14, p<$ .01 ; group $\times$ session: $F(8,32)=2.36, p>.05$ ]. On the test of retention (one session of six trials), 1 month after the last day of training, the performance of the two groups was comparable $[t(6)=0.29, p>.10]$.

\section{DISCUSSION}

The present results demonstrate that combined lesions of the hippocampus and amygdala in monkeys produce severe retrograde amnesia for information acquired between 2 weeks and 8 months prior to surgery. Performance was close to chance for the 100 preoperatively learned object pairs, and memory loss was equally severe across the five preoperative time periods in which learning occurred. Thus, marked retrograde amnesia was observed, and there was no evidence for a temporal gradient of retrograde amnesia. Finally, despite the severe retrograde amnesia for object pairs, memory for a preoperatively acquired motor skill was intact after surgery.

In an effort to detect memory for the preoperatively learned object pairs, we carried out four additional tests during the 8 months after surgery. On these tests, performance remained impaired despite our (1) repeating the retention test to assess the possibility of spontaneous recovery; (2) preceding the retention test with training on new object-discrimination problems, to ensure that the appropriate strategy was available to the animals during the retention test; (3) constructing a three-choice retention test (instead of two-choice), to increase test sensitivity; and (4) retraining a subset of the preoperatively learned object pairs, to determine if savings could be observed during relearning. In summary, bilateral medial temporal lesions produced retrograde amnesia covering at least 8 months with no evidence of a temporal gradient. Indeed, when all five postoperative retention tests are considered, the monkeys with medial temporal lesions performed at chance on 24 of the 25 time points.

Further studies are needed before general conclusions can be reached about the temporal features of retrograde amnesia following medial temporal lesions. For example, the absence of a temporal gradient in the present study could have been due to the fact that leaining was not scheduled long enough before surgery. Perhaps if object pairs had been learned longer than 8 months prior to surgery, memory for these object pairs would have survived surgery. One indication that the preoperative training period of 8 months was too short is that normal monkeys exhibited no forgetting during this interval. In previous studies of mice and humans, temporally limited retrograde amnesia was observed only in cases in which normal forgetting occurred during the period of observation (Cohen \& Squire, 1981; Squire, Chace, \& Slater, 1976; Squire \& Cohen, 1979; Squire, Slater, and Chace, 1975; Squire \& Spanis, 1984). For example, ECS produced retrograde amnesia for 1-day- to 3-week-old memories in mice, but not for older memories. Normal mice exhibited gradual forgetting between 1 day and 3 weeks after learning. To our knowledge, temporally graded retrograde amnesia has never been demonstrated in a case in which normal memory was unchanged across the time period containing the temporal gradient. (The remote memory impairment of patients with Korsakoff's syndrome is temporally graded [Albert, Butters, \& Levin, 1979; Cohen \& Squire, 1981]. However, part of this impairment reflects longstanding anterograde amnesia. What part of the impairment reflects retrograde amnesia and whether the retrograde portion of the impairment is temporally graded are not clear.)

Thus, it seems likely that if a study with monkeys is to provide a proper test of the nature of retrograde amnesia, normal animals should exhibit forgetting during the preoperative study period. Two ways to establish this condition would be to study memory across a time period longer than the 8 months of the present study and to reduce the number of learning trials given for each of the $20 \mathrm{ob}-$ ject pairs in each set. A third possibility is that the kind of task is important and that the retrograde amnesia results obtained with the object-discrimination task used here may differ from the results obtained with other tasks sensitive to medial temporal lesions.

In amnesic patients with known medial temporal lesions, recall of very old memories seems to be as good as in normal, intact subjects (Corkin, 1984; Marslen-Wilson \& Teuber, 1975; Zola-Morgan, Cohen, \& Squire, 1983; Zola-Morgan, Squire, \& Amaral, 1986). In experimental animals, retrograde amnesia following medial temporal lesions has been studied only infrequently (see Harley, 1979; Uretsky \& McCleary, 1969). Recently Staubli, Fraser, Kessler, and Lynch (1986) reported that bilateral entorhinal lesions in rats produced marked anterograde amnesia but had only a mild effect on preoperatively acquired information. The present study is the first to explore the temporal features of retrograde amnesia in monkeys. The results show that extensive retrograde amnesia can occur after medial temporal lesions, but many questions remain about its pattern and its temporal limits. Because the nature of retrograde amnesia is integral to notions about the role of the medial temporal region in normal memory function, additional investigations in experimental animals should be fruitful.

\section{REFERENCES}

AGgLeton, J. P. (1985). A description of intra-amygdaloid connections in Old World monkeys. Experimental Brain Research, 57, 390-399.

AlBert, M. S., Butters, N., \& Levin, J. (1978). Temporal gradients in the retrograde amnesia of patients with alcoholic Korsakoff's disease. Archives of Neurology, 36, 211.

BADDELEY, A. (1982). Implications of neuropsychological evidence for theories of normal memory. Philosophical Transactions of the Royal Society of London, B 298, 59-72.

BonIN, G. VON, \& BAILEY, P. (1947). The neocortex of Macaca mulatta. Urbana: University of Illinois Press.

BuTters, N. (1984). Alcoholic Korsakoff's syndrome: An update. Seminars in Neurology, 4, 226-244.

Cermak, L. S., \& O'Connor, M. (1983). The anterograde and retro- 
grade retrieval ability of a patient with amnesia due to encephalitis. Neuropsychologia, 21, 213-234.

Cohen, N. J., \& SQuire, L. R. (1981). Retrograde amnesia and remote memory impairment. Neuropsychologia, 19, 337-356.

CorkIn, S. (1984). Lasting consequences of bilateral medial temporal lobectomy: Clinical course and experimental findings in H.M. Seminars in Neurology, 4, 249-259.

Davis, R. T., McDowell, A. A., Deter, C. W., Steele, J. P. (1956). Performance of rhesus monkeys on selected laboratory tasks presented before and after a large single dose of whole body $\mathrm{x}$-radiation. Journal of Comparative \& Physiological Psychology, 49, 20-26.

GeLlerman, L. W. (1933). Chance orders of alternating stimuli in visual discrimination experiments. Journal of General Psychology, 42, 207-208.

HALGREN, E. (1984). Human hippocampal and amygdala recording and stimulation: Evidence for a neural model of recent memory. In L. R. Squire \& N. Butters (Eds.), The neuropsychology of memory (pp. 165182). New York: Guilford Press.

HARLEY, C. W. (1979). Arm choices in a sunburst maze: Effects of hippocampectomy in the rat. Physiology \& Behavior, 23, 283-290.

HARLOW, H. F. (1944). Studies in discrimination learning by monkeys: I. The learning of discrimination series and the reversal of discrimination series. Journal of General Psychiatry, 30, 3-12.

MahuT, H., \& Moss, M. (1984). Consolidation of memory: The hippocampus revisited. In L. R. Squire \& N. Butters (Eds.), Neuropsychology of memory (pp. 297-315). New York: Guilford Press.

Marslen-Wilson, W. D., \& Teuber, H. L. (1975). Memory for remote events in anterograde amnesia: Recognition of public figures from news photographs. Neuropsychologia, 13, 353-364.

Mishkin, M, (1978). Memory in monkeys severely impaired by combined but not by separate removal of amygdala and hippocampus. $\mathrm{Na}$ ture, 273, 297-298.

Mishkin, M. (1982). A memory system in the monkey. Philosophical Transactions of the Royal Society of London, B 298, 85-95.

NeLson, T. (1978). Detecting small amounts of information in memory: Savings for unrecognized items. Journal of Experimental Psychol ogy: Human Learning \& Memory, 4, 453-468.

Parkin, A. J. (1984). Amnesic syndrome: A lesion-specific disorder? Cortex, 20, 479-508.

Rose, F. C., \& Symonds, C. P. (1960). Persistent memory defect following encephalitis. Brain, 83, 195-212.

SChaCter, D. L. (1985). Multiple forms of memory in humans and animals. In N. M. Weinberger, J. L. McGaugh, \& G. Lynch (Eds.), Memory systems of the brain (pp. 351-379). New York: Guilford Press.

SCOVILLE, W. B., \& MILNER, B. (1957). Loss of recent memory after bilateral hippocampal lesions. Joumal of Neurology \& Psychiatry, 20, 11-21.

SQuiRe, L. R. (1984). ECT and memory dysfunction. In B. Leher, R. D. Weiner, \& R. H. Belmaker (Eds.), Basic mechanisms of ECT (pp. 156163). London: Libby.

SQuire, L. R. (1986). Mechanisms of memory. Science, 232, 1612-1619.

SQuire, L. R. (1987). Memory and brain. New York: Oxford University Press.

Squire, L. R., Chace, P. M., Slater, P. C. (1976). Retrograde amnesia following electroconvulsive therapy. Nature, 260, 775-777.

SQuIRE, L. R., \& CoHEN, N. J. (1979). Memory and amnesia: Resistance to disruption develops for years after learning. Behavioral \& Neural Biology, 25, 115-125.
SQuire, L. R., \& CoHen, N. J. (1984). Human memory and amnesia. In G. Lynch, J. L. McGaugh, \& N. M. Weinberger (Eds.), Neurobiology of learning and memory (pp. 3-64). New York: Guilford Press.

Squire, L. R., Cohen, N. J., \& Nadel, L. (1984). The medial temporal region and memory consolidation: A new hypothesis. In $\mathbf{H}$. Weingartner \& E. Parker (Eds.), Memory consolidation (pp. 185-210). Hillsdale, NJ: Erlbaum.

Squire, L. R., Slater, P. C., \& Chace, P. M. (1975). Retrograde amnesia: Temporal gradient in very long-term memory following electroconvulsive therapy. Science, 187, 77-79.

SQUIRE, L. R., \& SPANIS, C. W. (1984). Long gradient of retrograde amnesia in mice: Continuity with the findings in humans. Behavioral Neuroscience, $98,345-348$.

SQUIRE, L. R., \& ZoLA-MORGaN, S. (1983). The neurology of memory: The case for correspondence between the findings for man and nonhuman primate. In J. A. Deutsch (Ed.), The physiological basis of memory (2nd ed., pp. 199-268). New York: Academic Press.

Staubli, U., Fraser, D., Kessler, M., \& LynCh, G. (1986). Studies in retrograde and anterograde amnesia after hippocampal denervation by entorhinal lesions. Behavioral Neural Biology, 46, 432-444.

SzABO, J., CowAN, W. M. (1984). A stereotaxic atlas of the brain of the cynomolgus monkey (Macaca fascicularis). Joumal of Comparative Neurology, 222, 265-300.

TEYLER, T. J., \& DiSCENNA, P. (1986). The hippocampal memory indexing theory. Behavioral Neuroscience, 100, 147-154.

URETSKY, E., \& MCCLEARY, R. A. (1969). Effect of hippocampal isolation on retention. Journal of Comparative \& Physiological Psychology, 68, 1-8.

WEISKRANTZ, L. (1982). Comparative aspects of studies in amnesia. Philosophical transactions of the Royal Society of London, B 298 , 97-109.

WeISKRANTZ, L. (1985). On issues and theories of the human amnesic syndrome. In. N. M. Weinberger, J. L. McGaugh, \& G. Lynch (Eds.), Memory systems of the brain (pp. 380-415). New York: Guilford Press.

WICKELGREN, W. A. (1979). Chunking and consolidation: A theoretical synthesis of semantic networks, configuring in conditioning, S-R versus cognitive learning, normal forgetting, the amnesic syndrome, and the hippocampal arousal system. Psychological Review, 86, 44-60.

Zola-Morgan, S., Cohen, N. J., \& SQUiRe, L. R. (1983). Recall of remote episodic memory in amnesia. Neuropsychologia, 21, 487-500.

Zola-Morgan, S., \& SQUIRE, L. R. (1984). Preserved learning in monkeys with medial temporal lesions: Sparing of motor and cognitive skills. Joumal of Neuroscience, 4, 1072-1085.

Zola-Morgan, S., \& Seuire, L. R. (1985). Medial temporal lesions in monkeys impair memory in a variety of tasks sensitive to amnesia. Behavioral Neuroscience, 99, 22-34.

Zola-Morgan, S., Squire, L. R., Amaral, D. G. (1986). Human amnesia and the medial temporal region: Enduring memory impairment following a bilateral lesion limited to field CAl of the hippocampus. Journal of Neuroscience, 6, 2950-2967.

Zola-Morgan, S., Souire, L. R., Mishin, M. (1982). The neuroanatomy of amnesia: Amygdala-hippocampus vs. temporal stem. Science, 218, 1337-1339.

(Manuscript received August 8, 1986; revision accepted for publication January 5,1987 ). 